J. Lake Sci. (湖泊科学), 2015, 27 (4): 583-590

DOI $10.18307 / 2015.0404$

(c) 2015 by Journal of Lake Sciences

\title{
生源性湖泛水体主要含硫致臭物及其产生机制”
}

\author{
卢 信 ${ }^{1,2}$, 刘 成 ${ }^{2,3}$, 尹洪斌 ${ }^{2}$ 范成新 ${ }^{2 * *}$ \\ $(1:$ 江苏省农业科学院, 南京 210014) \\ (2: 中国科学院南京地理与湖泊研究所湖泊与环境国家重点实验室, 南京 210008) \\ (3: 中国科学院大学,北京 100049)
}

\begin{abstract}
摘 要: 由水生生物残体诱发的湖泛发生过程中水体高浓度的含硫致臭物质对水生态安全以及人类健康造成严重危害, 这些致臭物质的来源途径及其产生机制至今尚无详细报道. 通过使用藻类、浮游动物、鱼类、底栖动物以及水生植物等多 种生物残体进行了模拟实验, 结果发现各类生物残体均能导致湖泛的发生,其中藻类残体中含有的大量含硫氨基酸导致 水体挥发性有机硫化物浓度升高尤为突出. 使用蛋氨酸作为前驱物质进行模拟实验发现, 影响蛋氨酸降解的非生物因素 主要为光照和厌氧条件, 其中光照的影响更大. 对蛋氨酸降解率与铵态氮浓度变化的关系进行研究后发现, 无论有无生 物作用二者均有显著的相关性,表明蛋氨酸降解的第一步应为发生脱氨基作用. 对 $\alpha$-差丁酸、 $\alpha$-酮丁酸和 4-甲硫基-2-氧 代丁酸等中间产物在体系中的变化进行研究后发现,4-甲硫基-2-氧代丁酸在体系中很难积累,而 $\alpha$-差丁酸和 $\alpha$-酮丁酸则 有较明显的累积过程, 说明脱甲硫基作用可能在脱氨基作用后很短时间内发生,或二者同时发生, 由此产生甲硫醇, 甲硫 醇再通过各种途径转化为二甲基硫醚、二甲基二硫醚、二甲基三硫醚以及硫化氢等.
\end{abstract}

关键词: 湖泛;含硫氨基酸;蛋氨酸;挥发性硫化物;脱氨基作用;太湖;贡湖湾

\section{The main sulfur-containing odorous compounds and their forming mechanisms in waters during bio-induced black bloom}

\author{
LU Xin ${ }^{1,2}$, LIU Cheng ${ }^{2,3}$, YIN Hongbin ${ }^{2} \&$ FAN Chengxin ${ }^{2}$ \\ (1: Jiangsu Academy of Agricultural Sciences, Nanjing 210014, P. R. China) \\ (2: State Key Laboratory of Lake Science and Environment, Nanjing Institute of Geography and Limnology, Chinese Academy \\ of Sciences, Nanjing 210008, P. R. China) \\ (3: University of Chinese Academy of Sciences, Beijing 100049, P. R. China)
}

\begin{abstract}
High levels of sulfur-containing odorous compounds can always be detected in waters during black bloom induced by aquatic debris. These contaminants are great threatens to water ecological security and human health. However, detailed reports focused on the sources and forming mechanisms of these odorous compounds were seldom seen. In this study, various aquatic debris like algae, zooplankton, fishes, benthic fauna and aquatic plants were used to simulate the occurrence of black bloom. Results showed that black bloom can be triggered by all of these aquatic debris. The highest levels of volatile organic sulfur compounds were detected in the added-algae treatments because of the high content of sulfur-containing amino acids in algae. Methionine was used as precursor of VOSCs during the experiment. Illumination and anaerobic conditions, especially illumination, were found to be more favorable for the degradation of methionine. Significant correlations were found between the degradation rate of methionine and the ammonium nitrogen levels, demonstrating that the first step of methionine degradation was deamination. The variations of several intermediate products ( $\alpha$-hydroxybutyric acid, $\alpha$-oxobutyric acid and 4-methylthio-2-oxobutyric acid (KMBA)) in the degradation system were studied. The accumulation of KMBA was quite low compared to that of $\alpha$-hydroxybutyric acid and $\alpha$-oxobutyric acid. These results demonstrated that the demethylthio reaction happened with a short period of time after deamination. Or may-
\end{abstract}

* 中国科学院南京地理与湖泊研究所“一三五”战略发展规划项目 (NIGLAS2012135008) 和江苏省太湖水污染治理 专项 ( TH2013214) 联合资助. 2015-01-14 收稿;2015-04-13 收修改稿. 卢信 (1978 ), 女, 博士, 助理研究员; E-mail:lxdeng@126.com.

** 通信作者; E-mail: cxfan@ niglas. ac.cn. 
be these two reactions happened at the same time. Methanethiol was generated during this period and then transformed to dimethyl sulfide, dimethyl disulfide, dimethyl trisulfide and $\mathrm{H}_{2} \mathrm{~S}$ through a variety of ways.

Keywords: Black bloom; sulfur-containing amino acids; methionine; volatile sulfur compounds; deamination; Lake Taihu; Gonghu Bay

湖泛是近二十年来常见于我国浅水湖泊的一种极端水环境问题 ${ }^{[1-4]}$, 在我国太湖、巢湖等重要湖泊中均 曾有发现. 2007 年, 由于湖泛造成自来水变色发臭, 导致无锡市居民饮用水安全受到严重威胁, 也使得湖泛 问题成为政府、学界及公众关注的焦点 ${ }^{[2,5]}$.

湖泛发生时水体通常呈现的共同特征有 ${ }^{[6-8]}$ : 极低的溶解氧 (DO) 浓度 (接近于 $0 \mathrm{mg} / \mathrm{L}$ )、极低的氧化还 原电位 $(\mathrm{Eh})$ 、较低的 $\mathrm{pH}$ 值、高营养盐负荷以及水体发黑发臭等. 对于水体发黑的主要原因, 研究者 ${ }^{[9-10]}$ 认为 金属硫化物 (主要为铁硫化物, 如 FeS 等) 在上覆水体中的累积导致水体颜色逐渐变深并最终发黑. 湖泛发 生前通常会经历 DO 和 $\mathrm{Eh}$ 的迅速降低, 在这一过程中, $\mathrm{Fe}^{2+}$ 和 $\Sigma \mathrm{H}_{2} \mathrm{~S}$ 在沉积物一水界面处迅速累积并形成 $\mathrm{FeS}$ 等致黑物质 ${ }^{[6]}$, 因此湖泛发生过程中水体通常自下而上逐渐变黑, 这一过程短则数小时, 长则数天. 水体 变黑通常是判断湖泛发生的主要标志,而水体发臭难以去除可能对饮用水安全造成更大的威胁.

已有对野外水体湖泛发生过程中致臭物质的研究表明, 无论是藻源性 ${ }^{[2]}$ 还是草源性 ${ }^{[7]}$ 湖泛, 主要致臭 物质可能为嗅味物质, 包括土腥素 (Geosmin)、二甲基异茨醇 ( MIB) 和 2-甲氧基-3-异丁基吡嗪 ( IBMP 等), 及 挥发性硫化物 (volatile sulfur compounds, VSCs), 包括硫化氢 $\left(\mathrm{H}_{2} \mathrm{~S}\right.$ )、二甲基硫醚 (dimethyl sulfide, DMS) 、二 甲基二硫醚（dimethyl disulfide, DMDS)、二甲基三硫醚（dimethyl trisulfide, DMTS) 和甲硫醇 (methanethiol, MTL) 等, 其中尤以 MTL、DMS、DMDS 和 DMTS 等挥发性有机硫化物 (VOSCs) 为主. 然而, 这些致臭物质的主 要来源及其产生机制至今仍无较为详尽的报道. 前期研究 ${ }^{[11]}$ 已表明, 有机质对水体发黑具有重要诱导作用, 然而, 只有含硫有机物才具有致臭作用, 且含硫氨基酸为湖泛发生过程中 VOSCs 的主要前驱物质. 由于许多 水生生物体内都含有一定量的含硫氨基酸, 而湖泛发生时 VOSCs 的前驱物含硫氨基酸是否来源于这些水生 生物, 又是通过怎样的途径进行转化, 至今尚未见报道. 因此, 本文在野外湖泛监测采样的基础上, 研究了不 同水生生物诱导下湖泛的发生情况, 并以蛋氨酸为前驱物质, 通过设置不同的降解体系探讨含硫氨基酸代 谢产生 VOSCs 的可能途径, 以期进一步揭示生源性湖泛发生过程中水体主要致臭物的产生机制.

\section{1 材料与方法}

\section{1 野外湖泛监测与采样}

本研究 3 个调查采样点 $\left(1^{\prime \prime} 31^{\circ} 23^{\prime} 11^{\prime \prime} \mathrm{N}, 120^{\circ} 17^{\prime} 44^{\prime \prime} \mathrm{E} ; 2^{*} 31^{\circ} 26^{\prime} 6^{\prime \prime} \mathrm{N}, 120^{\circ} 22^{\prime} 37^{\prime \prime} \mathrm{E} ; 3^{\#} 31^{\circ} 21^{\prime} 12^{\prime \prime} \mathrm{N}, 120^{\circ} 14^{\prime}\right.$ $\left.29^{\prime \prime} \mathrm{E}\right)$ 均分布在太湖贡湖湾内, 是 2007 年太湖发生水危机事件的区域. 除监测主要致臭物质外, 同时还分别 监测 3 个点位的水体基本理化性质和营养盐浓度. 分别于 2010 年 5-9 月每月中旬 (该段时间通常为太湖蓝 藻聚集密度较高的季节) 在 3 个采样点采集 $500 \mathrm{ml}$ 水样,采集水样时确保水流益出瓶口, 迅速用螺口螺紧. 同时采集 $500 \mathrm{ml}$ 水样用于测定总磷 $(\mathrm{TP})$ 、总氮 $(\mathrm{TN})$ 、铵态氮 $\left(\mathrm{NH}_{4}^{+}-\mathrm{N}\right)$ 、磷酸盐 $\left(\mathrm{PO}_{4}^{3-}-\mathrm{P}\right)$ 和叶绿素 $\mathrm{a}(\mathrm{Chl} . \mathrm{a})$ 浓度. 现场应用水质速测仪 (YSI) 测定水体的 $\mathrm{pH}$ 、氧化还原电位 ( Eh) 以及溶解氧 (DO). 所采集样品放人冷 藏保温箱内保存,于 $4 \mathrm{~h}$ 内带回实验室进行分析.

\section{2 模拟实验采样点及样品采集}

采样点选择在太湖月亮湾水域 $\left(31^{\circ} 24^{\prime} 35.8^{\prime \prime} \mathrm{N}, 120^{\circ} 6^{\prime} 4.6^{\prime \prime} \mathrm{E}\right)$, 其水域面积为 $10.51 \mathrm{~km}^{2}$, 位于太湖北部 梅梁湾与竺山湾间, 是太湖典型的蓝藻堆积区域和湖泛易发区域. 使用柱状采样器 $(\phi 90 \mathrm{~mm} \times 500 \mathrm{~mm})$ 在 该区域采集无扰动柱状样品,同时采集上覆水用于室内模拟实验.

\section{3 实验设计}

1.3.1 生源性湖泛模拟 由于生物残体中含有大量含硫氨基酸, 因此, 为了验证生源性湖泛发生过程中致臭 物质的产生与生物残体的关系, 在室内使用 $\mathrm{Y}$ 型沉积物再悬浮发生装置 ${ }^{[12]}$ 模拟多种生物堆积下湖泛的发 生情况. 主要实验组有: 未添加生物残体的对照组、添加 $5000 \mathrm{~g} / \mathrm{m}^{2}$ 太湖鲜藻组、添加 $1000 \mathrm{~g} / \mathrm{m}^{2}$ 大型浮游动 物残体组 (主要是枝角类和桡足类, 使用 $13^{\#}$ 浮游生物网, 以“8”字型从水体中捞取)、添加 $5000 \mathrm{~g} / \mathrm{m}^{2}$ 底栖动 
物残体组 (将去壳后的河蚬残体剪碎加人)、添 加 $5000 \mathrm{~g} / \mathrm{m}^{2}$ 鱼类残体组 (将太湖梅鲚鱼残体捣 碎后加人) 以及添加 $5000 \mathrm{~g} / \mathrm{m}^{2}$ 水生植物残体组 (将太湖蕰草剪碎后加人). 温度设置为 $28^{\circ} \mathrm{C}$, 水深为 $1.8 \mathrm{~m}$, 接近太湖平均水深 ${ }^{[13]}$. 观察湖泛 是否发生并检测湖泛发生时水体中致臭物质 浓度.

1.3.2 非生物因素对蛋氨酸降解生成致臭物的 影响 将表层 $0 \sim 6 \mathrm{~cm}$ 原位沉积物充分混匀后 称取 $600 \mathrm{~g}$ 放人具有气密性的密闭反应系统中 (图 1 $)^{[11]}$,将原位采集的湖水过滤去除悬浮颗 粒物之后配制成 $1.0 \mathrm{~g} / \mathrm{L}$ 的蛋氨酸溶液, 于 $121^{\circ} \mathrm{C}$ 下灭菌 $40 \mathrm{~min}$ 后加人上述反应系统中. 共设置 3 个处理:好氧 $(\mathrm{DO}>6 \mathrm{mg} / \mathrm{L})$ 光照、厌 氧 ( DO $<0 \mathrm{mg} / \mathrm{L})$ 光照和戻氧避光, 定时取水 样测定 VOSCs 浓度, 以研究氧气与光照等非生

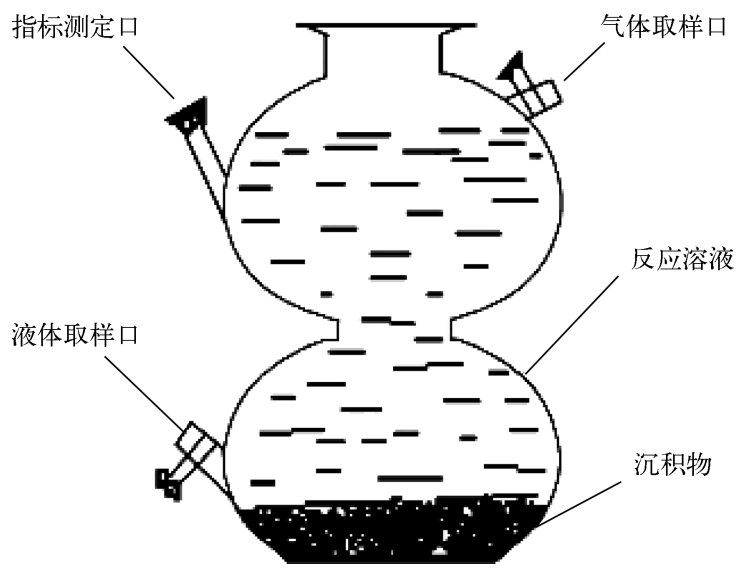

图 1 密闭反应系统示意图

Fig. 1 Schematic diagram of the airtight reaction system 物因素对含硫氨基酸降解生成 VOSCs 的影响.

1.3. 3 不同降解体系中含硫氨基酸的降解过程 向反应系统中加人不同溶液设置 5 个实验组: 对照, 高温高 压灭菌 $\left(121^{\circ} \mathrm{C}\right.$ 灭菌 $\left.60 \mathrm{~min}\right)$, 添加 $2.0 \mathrm{mmol} / \mathrm{L}$ 铇酸钠作为硫酸盐还原菌 $(\mathrm{SRB})$ 抑制剂, 添加 $5.0 \mathrm{mmol} / \mathrm{L}$ 溴 乙基磺酸钠 (BES) 作为产甲烷菌抑制剂, 添加 $50 \mathrm{ml} \mathrm{SRB118}$ 菌液 ${ }^{[14]}$.

通过研究含硫氨基酸降解过程中 $\mathrm{NH}_{4}^{+}-\mathrm{N}$ 以及 $\alpha$-酮丁酸、 $\alpha$-羊丁酸、4-甲硫基-2-氧代丁酸 $(\mathrm{KMBA}$ ) 等中 间产物的生成情况, 研究含硫氨基酸的降解过程.

\section{4 样品分析}

野外采集水样中致臭物的测定采用顶空-固相微萃取 (HS-SPME)-GC-MS (Agilent1790-5975C) 系统组合 完成. 测试方法及条件详见文献 [15]; 水体中的 TN 和 TP 浓度均采用过硫酸钾氧化-比色法测定; $\mathrm{NH}_{4}^{+}-\mathrm{N}$ 和 $\mathrm{PO}_{4}^{3-}-\mathrm{P}$ 浓度均采用 Skalar 流动分析仪测定; Chl. a 浓度采用丙酮提取一分光光度法分析.

蛋氨酸采用亚硝基铁氭化钾-可见分光光度法测定 ${ }^{[16]}$; 水样中挥发性硫化物分析由顶空固相微萃取 (HS-SPME) 预浓缩系统和 Agilent 7890A/5973N GC-MS(安捷伦公司, 美国) 系统组合完成; 气体样品的分析 使用气相色谱一火焰光度检测器 Agilent 7890A GC-FPD 完成,测试方法及条件详见文献[11].

蛋氨酸代谢中间产物采用 Agilent 1200 HPLC 测定, 仪器配置及方法如下: 检测器为二极管阵列检测器 (DAD), 色谱柱采用 Agilent Eclipse XDB-C18 (固定相液膜厚度 $5 \mu \mathrm{m}$, 内径 $4.6 \mathrm{~mm} \times$ 长度 $150 \mathrm{~mm}$ ), 配自动进样 器. 以 $0.02 \mathrm{~mol} / \mathrm{L}$ 的 $\mathrm{KH}_{2} \mathrm{PO}_{4}$ 缓冲盐水溶液 ( $\mathrm{A}$ 相) 和乙腈为流动相 ( $\mathrm{B}$ 相), 采用梯度洗脱的方式由初始比例 $\mathrm{A}: \mathrm{B}$ 为 95:5 经 $15 \mathrm{~min}$ 洗脱至 $\mathrm{A}: \mathrm{B}$ 为 80:20, 恒定流速为 $1 \mathrm{ml} / \mathrm{min}$, 检测波长为 $215 \mathrm{~nm}$, 柱温为 $30^{\circ} \mathrm{C}$, 进样量为 $20 \mu \mathrm{l}$.

\section{2 结果与讨论}

\section{1 湖泛易发水域主要致臭物及水体基本理化参数}

从野外采集的实际样品看, 太湖贡湖湾 3 个采样点水体中的 Geosmin、MIB 和 DMTS 是主要致臭物质, 其 分布具有一定的季节变化规律, 在蓝藻暴发初期其浓度处于一个相对较低的水平, 而在蓝藻暴发中后期部 分蓝藻处于衰亡阶段时,其浓度则相对较高 (图 2).

从各监测点位检测到的致臭物浓度变化范围来看, 与 Yang 等 ${ }^{[2]}$ 在 2007 年的测定值有较大偏差, 其在 贡湖湾水体中测得的主要致臭物为 DMTS, 浓度最高可达 $11399 \mathrm{ng} / \mathrm{L}$, 而在本研究中 Geosmin、MIB 和 DMTS 均为野外样品主要致臭物质,其中 DMTS 浓度最大只有 $10.1 \mathrm{ng} / \mathrm{L}$, 如此大的偏差反映出两者之间的形成机 制存在显著差别. Yang 等 ${ }^{[2]}$ 推断在 2007 年水危机中的高浓度 DMTS 是由未知的大型污水团造成的,不排 除外来污染的因素. 而本研究中的 DMTS 则有可能只是蓝藻暴发后在局部小区域造成厌氧, 从而引起局部 
口DMTS $\square$ Geosmin $\quad$ MIB
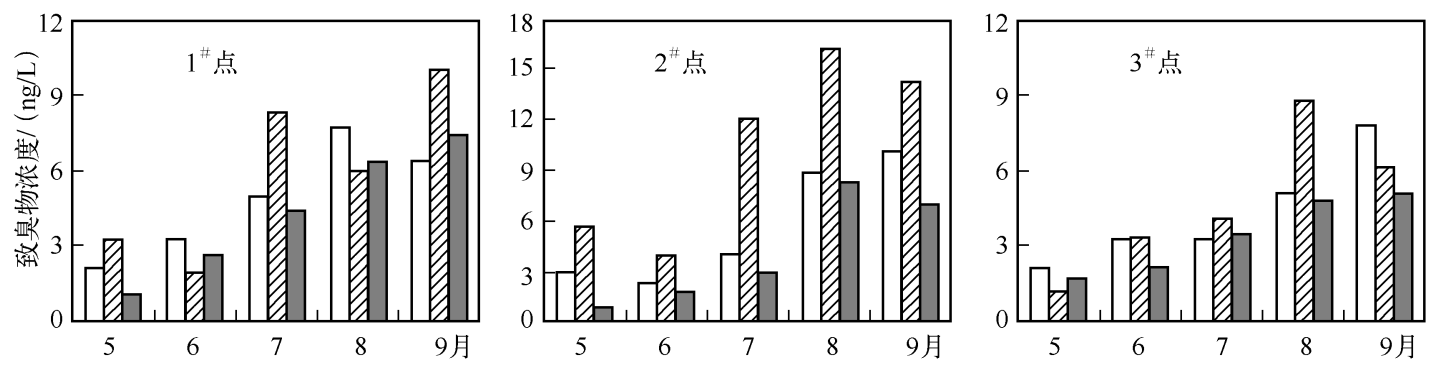

图 2 太湖贡湖湾 3 个采样点主要致臭物浓度的季节变化

Fig. 2 Seasonal changes of the main odorous substances concentrations of the three sampling sites in Gonghu Bay of Lake Taihu

小区域蓝藻逆境生长甚至死亡. 由于蓝藻细胞自身含有大量的 Geosmin 和 MIB, 在蓝藻暴发死亡的初期, 藻 细胞在极度戻氧条件下死亡破裂后其胞内嗅味物质会大量进人水溶液, 造成溶解性嗅味物质浓度升高 ${ }^{\left[{ }^{[17]}\right.}$; 随着藻体进一步降解, 其中所含的含硫氨基酸发生分解产生挥发性硫化物, 但由于小分子的挥发性硫化物 (如 $\mathrm{H}_{2} \mathrm{~S} 、$ MTL、DMS 和 DMDS) 的饱和蒸汽压较大, 挥发性比较强, 野外采样难以捕捉到, 因此只有挥发性低 的 DMTS 检出. 由于太湖是一个大型开放水体, 因此小区域内各种化学或生物过程同时并存, 各阶段的产物 也因此混合在一起.

各监测点位水体基本理化性质和主要营养盐参数的季节变化见表 1. 监测区域中的 TP、TN 以及其它水体 参数 (如 $\mathrm{pH} 、 \mathrm{Eh} 、 \mathrm{DO}$ 以及 $\mathrm{Chl} . \mathrm{a}$ 等) 与往年太湖水体中的浓度 ${ }^{[34]}$ 并无太多差异, 维持在一个正常的水平.

表 1 太湖贡湖湾水体基本理化性质和营养盐浓度变化

Tab. 1 The changes of basic physical-chemical properties and nutrient concentrations of water in Gonghu Bay of Lake Taihu

\begin{tabular}{|c|c|c|c|c|c|c|c|c|}
\hline & $\begin{array}{c}\mathrm{TN} / \\
(\mathrm{mg} / \mathrm{L})\end{array}$ & $\begin{array}{c}\mathrm{TP} / \\
(\mathrm{mg} / \mathrm{L})\end{array}$ & $\begin{array}{l}\mathrm{NH}_{4}^{+}-\mathrm{N} / \\
(\mathrm{mg} / \mathrm{L})\end{array}$ & $\begin{array}{c}\mathrm{PO}_{4}^{3-}-\mathrm{P} / \\
(\mathrm{mg} / \mathrm{L})\end{array}$ & $\mathrm{pH}$ & $\mathrm{Eh} / \mathrm{mV}$ & $\begin{array}{c}\mathrm{DO} / \\
(\mathrm{mg} / \mathrm{L})\end{array}$ & $\begin{array}{l}\text { Chl. a/ } \\
(\mathrm{mg} / \mathrm{L})\end{array}$ \\
\hline 5 月 & $0.76 \sim 2.11$ & $0.07 \sim 0.11$ & $0.21 \sim 0.87$ & $0.010 \sim 0.020$ & $7.78 \sim 7.90$ & $206 \sim 308$ & $5.76 \sim 6.43$ & $0.007 \sim 0.010$ \\
\hline 6 月 & $0.98 \sim 1.54$ & $0.03 \sim 0.09$ & $0.13 \sim 1.01$ & $0.008 \sim 0.020$ & $7.88 \sim 8.65$ & $327 \sim 354$ & $6.03 \sim 6.76$ & $0.008 \sim 0.010$ \\
\hline 7 月 & $1.02 \sim 1.87$ & $0.04 \sim 0.18$ & $0.45 \sim 1.32$ & $0.006 \sim 0.010$ & $7.75 \sim 8.76$ & $321 \sim 387$ & 7. $09 \sim 8.32$ & $0.010 \sim 0.013$ \\
\hline 8 月 & $1.33 \sim 3.21$ & $0.09 \sim 0.21$ & $0.54 \sim 1.87$ & $0.010 \sim 0.030$ & $8.32 \sim 8.36$ & $198 \sim 297$ & 4. $38 \sim 6.25$ & $0.020 \sim 0.030$ \\
\hline 9 月 & $1.42 \sim 3.21$ & $0.08 \sim 0.22$ & $0.87 \sim 2.01$ & $0.008 \sim 0.040$ & $8.56 \sim 8.55$ & $167 \sim 302$ & 4. $76 \sim 7.21$ & $0.018 \sim 0.021$ \\
\hline 平均值 & 1.45 & 0.23 & 0.48 & 0.02 & 8.32 & 242 & 5.87 & 0.02 \\
\hline
\end{tabular}

监测区水体的 $\mathrm{TP} 、 \mathrm{NH}_{4}^{+}-\mathrm{N}$ 以及 $\mathrm{Chl}$. a 与水体中的主要致臭物质呈显著或极显著关系 (表 2), 说明 这些因素可能是影响水体中致臭物质浓度分布的主要因素, 在蓝藻暴发、死亡期间, 蓝藻不但释放出 代谢物质 (如土腥素和二甲基异茨醇) 和一些营养盐 (如 $\mathrm{NH}_{4}^{+}-\mathrm{N}$ ), 同时还会增加水体的悬浮颗类物浓 度, 导致水体 TP 和 Chl. a 浓度增加. 有关这些致臭物质的产生机理需要进一步研究.

表 2 太湖贡湖湾水体主要异味物质与基本理化参数的相关性

Tab. 2 The correlation between basic physical-chemical parameters and main odor substances of water in Gonghu Bay of Lake Taihu

\begin{tabular}{ccccccccc}
\hline 异味物质 & $\mathrm{TN}$ & $\mathrm{TP}$ & $\mathrm{NH}_{4}^{+}-\mathrm{N}$ & $\mathrm{PO}_{4}^{3-}-\mathrm{P}$ & $\mathrm{pH}$ & $\mathrm{Eh}$ & $\mathrm{DO}$ & $\mathrm{Chl} . \mathrm{a}$ \\
\hline Geosmin & 0.121 & $0.231^{*}$ & $0.201^{*}$ & 0.114 & 0.009 & 0.012 & 0.021 & $0.341^{* *}$ \\
MIB & 0.213 & $0.321^{* *}$ & $0.320^{* *}$ & 0.080 & 0.017 & 0.016 & 0.023 & $0.301^{* *}$ \\
DMTS & 0.141 & $0.297^{* *}$ & $0.232^{*}$ & 0.156 & 0.007 & 0.013 & 0.125 & $0.425^{* *}$ \\
\hline
\end{tabular}

*表示显著相关, $P<0.05 ; * *$ 表示极显著相关,$P<0.01$. 


\section{2 不同生物残体对湖泛发生及致臭物质产生的影响}

前期研究 ${ }^{[11]}$ 已表明,有机质对水体发黑 具有重要诱导作用, 然而, 只有含硫有机物才 具有致臭作用, 且含硫氨基酸为湖泛发生过 程中 VOSCs 的主要前驱物质. 由于许多水生 生物体内都含有一定量的含硫氨基酸, 而湖 泛发生时 VOSCs 的前驱物含硫氨基酸是否来 源于这些水生生物, 又是通过怎样的途径转 化,至今尚未见报道. 本研究发现, 添加不同 生物残体后,水体均发生不同程度的湖泛. 各 实验组水体中均检出 DMS、DMDS 和 DMTS 等 主要湖泛致臭物质,且均超出各物质的嗅阈 值 (DMS、DMDS 和 DMTS 的嗅阈值分别为 2. 5、0.1 和 $10 \mathrm{ng} / \mathrm{L})^{[18]}$. 可见,生源性湖泛发 生过程中 VOSCs 的产生与水生生物有着重要 关联. 添加藻类时产生的 VOSCs 浓度相对高

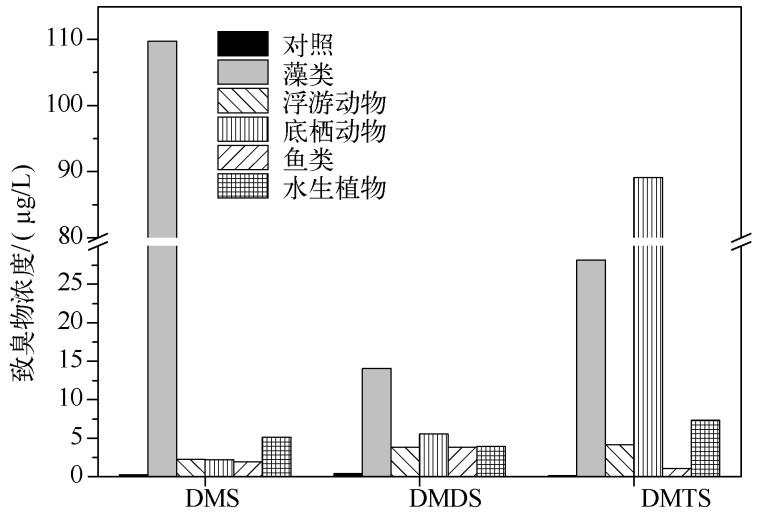

图 3 不同生源湖泛发生时水体致臭物浓度

Fig. 3 Concentrations of odorous compounds during the black blooms induced by various organisms 于其他实验组 (图 3). 其原因一方面是藻类代谢过程中会产生 $\operatorname{VOSC}^{[19-20]}$, 另一方面是太湖蓝藻中含硫氨基 酸浓度较高 ${ }^{[21]}$, 因而藻类死亡降解过程中也会产生大量的 VOSCs. 虽然底栖动物及水生植物残体产生的一些 硫化物浓度也较高, 但与蓝藻相比, 这些生物在湖泊水体中大量聚集并死亡的概率极小, 因此可以推断藻源性 湖泛是太湖最为常见的一种湖泛, 藻体中大量的含硫氨基酸导致湖泛发生时水体 VOSCs 浓度激增 ${ }^{[2]}$.

\section{3 非生物因素对含硫氨基酸降解的影响}

在生源性湖泛发生过程中, 氧气、光照等非生物因素均可能对含硫氨基酸降解为 VOSCs 的过程产生影 响. 不同氧气及光照条件下蛋氨酸降解生成 VOSCs, 其浓度随时间变化情况 ( 图 4) 表明, 厌氧及光照均能促 进蛋氨酸非生物降解生成 VOSCs, 其中 DMDS 与 MTL 为非生物降解产生的主要 VOSCs 组分, 也有少量 DMTS 和 DMS 产生, 但并未检测到 $\mathrm{H}_{2} \mathrm{~S}$. 同为光照条件下, 厌氧分解产生的 DMDS 和 MTL 为好氧分解的 $2 \sim 3$ 倍, 而同为厌氧条件下, 光照产生的 DMDS 和 MTL 最高浓度分别为 6.78 和 $6.44 \mu \mathrm{g} / \mathrm{L}$, 比避光条件下 DMDS $(1.32 \mu \mathrm{g} / \mathrm{L})$ 和 $\operatorname{MTL}(0.53 \mu \mathrm{g} / \mathrm{L})$ 浓度高 $5 \sim 10$ 倍左右. 可见, 在蛋氨酸非生物降解过程中, 光降解是非常重 要的一个因素, 厌氧也是促使蛋氨酸降解转化为 VOSCs 的重要原因之一, 但光照条件的影响大于氧气条件 的影响.

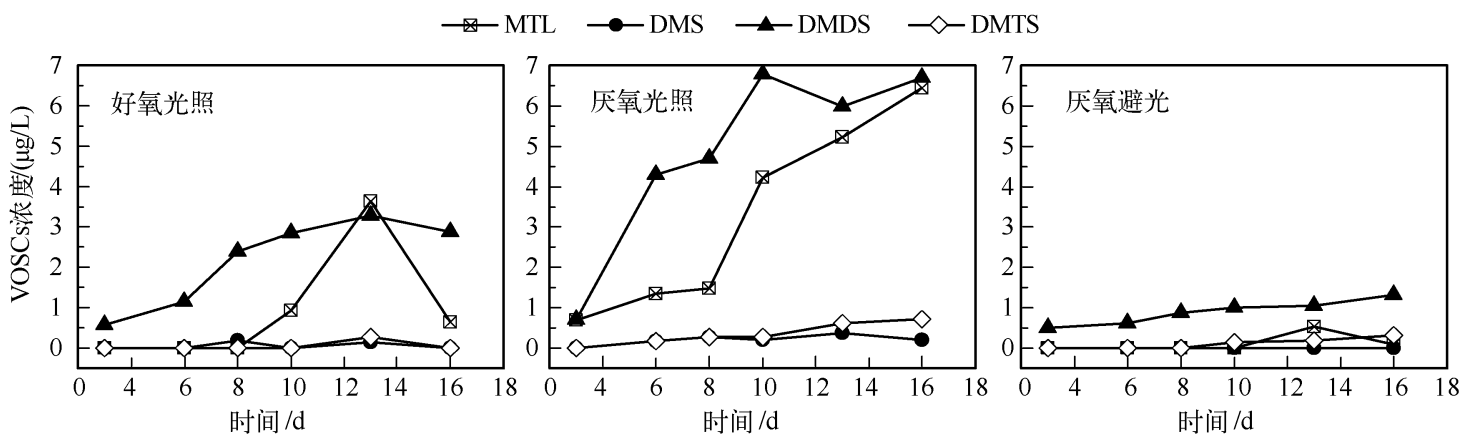

图 4 氧气及光照条件对蛋氨酸非生物降解生成 VOSCs 的影响

Fig. 4 The production of VOSCs from methionine degradation influenced by oxygen and illumination conditions

\section{4 不同降解体系中含硫氨基酸的降解过程}

从不同蛋氨酸降解体系中 $\mathrm{NH}_{4}^{+}-\mathrm{N}$ 浓度的变化情况来看 (图 5), 添加 $\mathrm{SRB}$ 菌液的处理 $\mathrm{NH}_{4}^{+}-\mathrm{N}$ 浓度最高, 


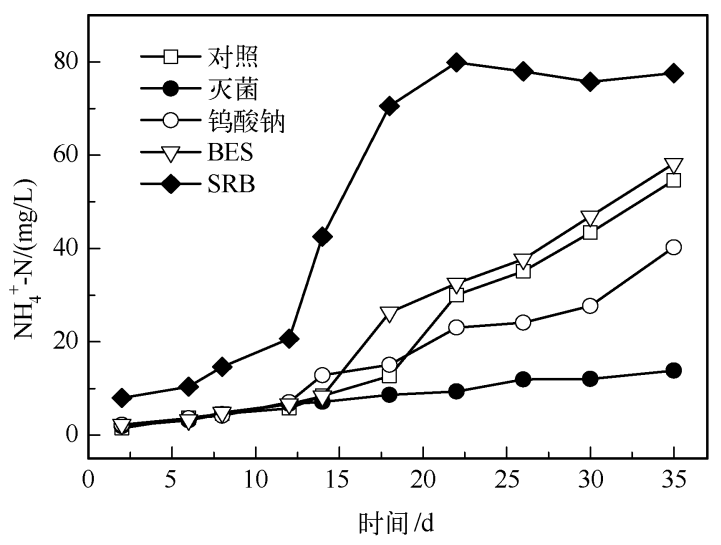

图 5 不同蛋氨酸降解体系中 $\mathrm{NH}_{4}^{+}-\mathrm{N}$ 浓度变化

Fig. 5 Variations of $\mathrm{NH}_{4}^{+}-\mathrm{N}$ concentrations under different methionine degradation treatments

其后依次为添加 BES(甲烷菌抑制剂) 处理、对照 处理、添加铇酸钠 (SRB 抑制剂) 处理和灭菌处 理. 对各处理组 $\mathrm{NH}_{4}^{+}-\mathrm{N}$ 浓度与相应的蛋氨酸降 解率进行相关性分析后发现 (图 6), 各处理组 蛋氨酸降解率均与 $\mathrm{NH}_{4}^{+}-\mathrm{N}$ 浓度呈极显著相关 $(P<0.01)$, 表明生物或非生物作用下, 蛋氨酸 降解的第一步会发生脱氨基作用或脱甲硫基作 用形成 $\mathrm{NH}_{4}^{+}-\mathrm{N} 、 \alpha$-羟丁酸、 $\alpha$-酮丁酸和 4-甲硫 基-2-氧代丁酸等. 而从各组 $\mathrm{NH}_{4}^{+}-\mathrm{N}$ 浓度变化来 看, 添加 SRB 菌液的处理组 $\mathrm{NH}_{4}^{+}-\mathrm{N}$ 浓度与其他 实验组间呈极显著相关 $(P<0.01)$, 说明 SRB 在 含硫氨基酸降解过程中有着重要的作用, 这也与 前期研究所发现的 SRB 对湖泛的重要作用 相符 ${ }^{[14]}$.
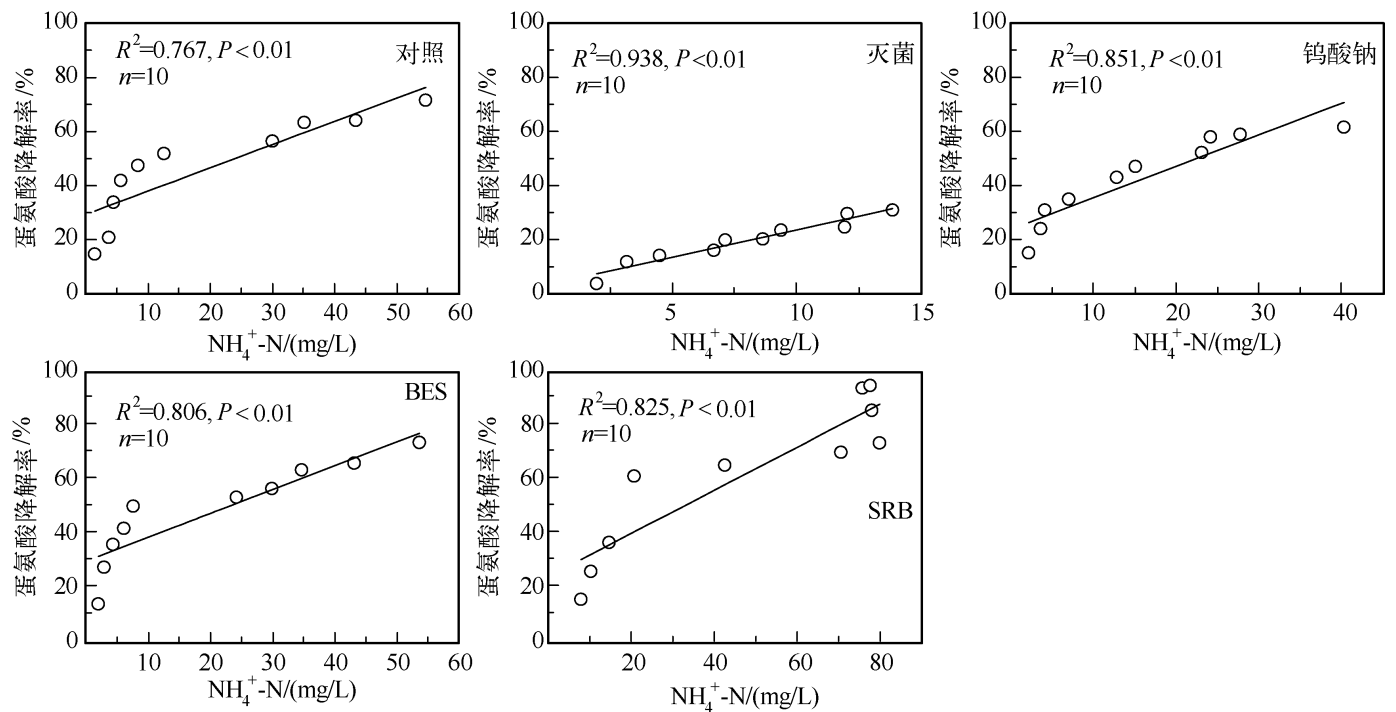

图 6 不同处理组中 $\mathrm{NH}_{4}^{+}-\mathrm{N}$ 浓度与蛋氨酸降解率的相关关系

Fig. 6 Correlations between $\mathrm{NH}_{4}^{+}-\mathrm{N}$ concentration and methionine degradation rate under different treatments

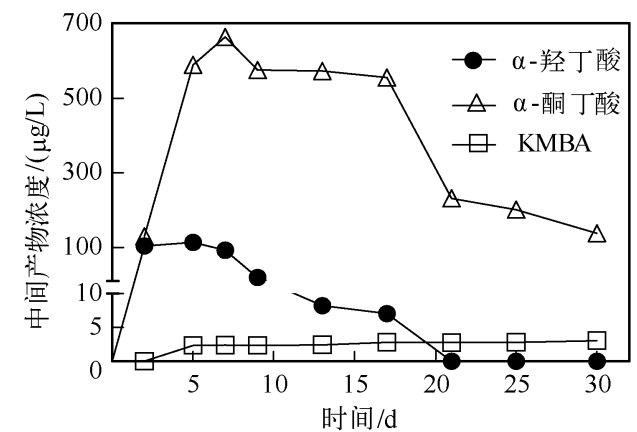

图 7 蛋氨酸降解主要中间产物浓度变化

Fig. 7 Dynamics of three intermediates concentration during the metabolism of methionine

\section{5 含硫氨基酸代谢产生致臭物的可能途径分析}

为探讨蛋氨酸降解的途径, 除对 $\mathrm{NH}_{4}^{+}-\mathrm{N}$ 和 VOSCs 等进行监测外, 还需对含硫氨基酸降解过程中产生的 $\alpha-$ 差了酸、 $\alpha$-酮丁酸和 KMBA 等中间产物动态变化进行研 究. $\alpha$-酮丁酸在实验开始后浓度即迅速升高, 并于 $7 \mathrm{~d}$ 内 达到最高值, 之后随着进一步降解而逐渐降低. 与 $\alpha$-酮 丁酸相似, $\alpha$-差弪丁酸在实验进行 $5 \mathrm{~d}$ 后浓度达到最高, 随 后浓度迅速降低并最终完全消失, 但其浓度要比 $\alpha$-酮丁 酸低得多(图 7). 而 KMBA 的浓度则始终保持在较低水 平 $(0 \sim 3.1 \mathrm{mg} / \mathrm{L})$, 其原因一方面可能是由于 KMBA 会 快速发生脱甲硫基作用转化为 MTL, 造成体系中 KMBA 无法大量累积; 另一方面可能是蛋氨酸除发生脱氨基作 
用生成 KMBA 外, 还可同时发生脱氨基作用和脱甲硫基作用,直接一步生成 MTL、 $\alpha$-酮丁酸和氨, 由此导致 体系中 KMBA 浓度较低而 $\alpha$-羟丁酸和 $\alpha$-酮丁酸却有较明显的累积过程.

根据本文的研究结果以及前期的一些研究 ${ }^{[1,22]}$, 推测蛋氨酸降解生成 VOSCs 的可能途径如图 8 所示.

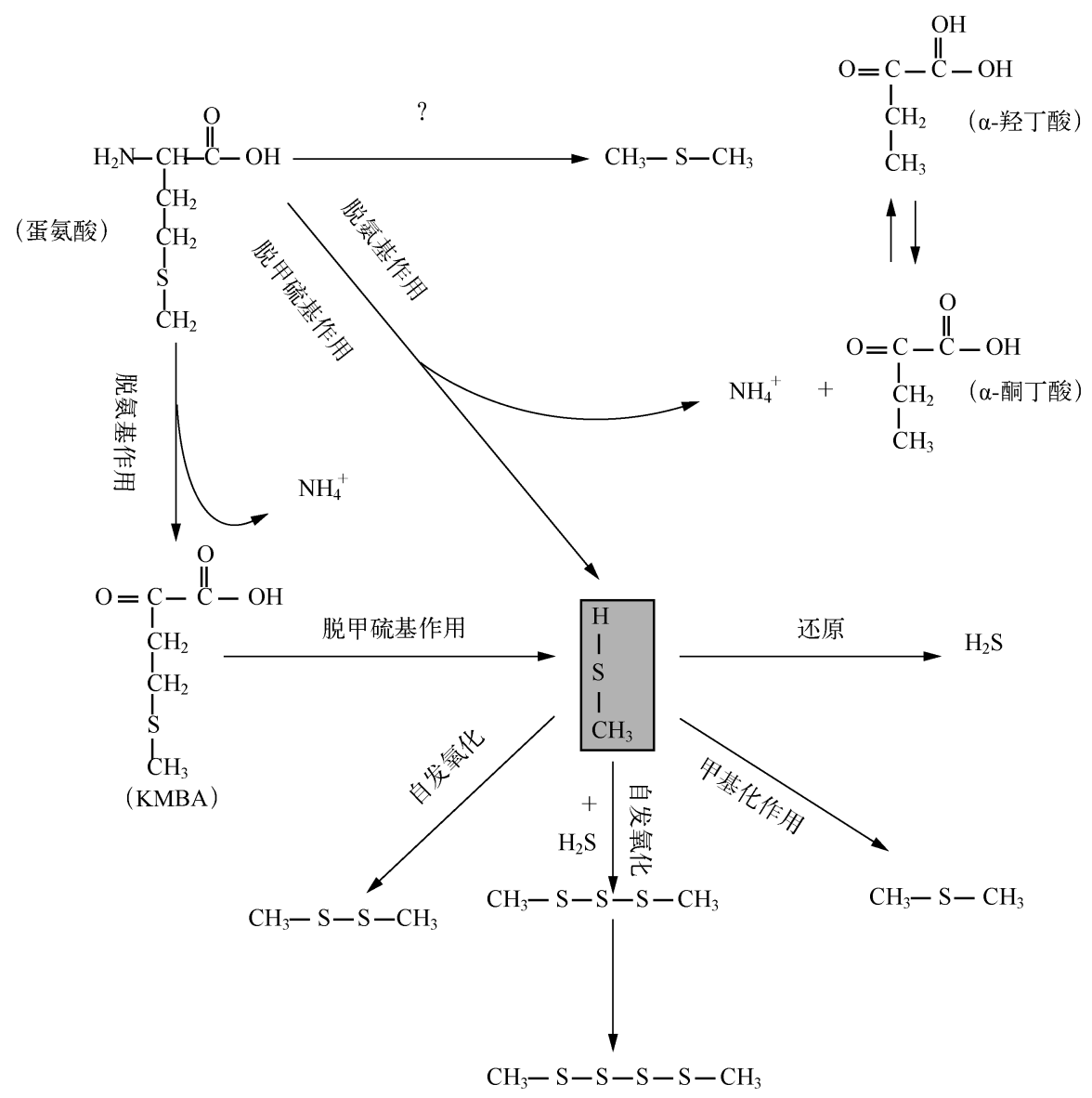

图 8 蛋氨酸降解为 VOSCs 的可能途径

Fig. 8 Proposed pathways of the catabolism of methionine to VOSCs

\section{3 结论}

在室内模拟条件下对生源性湖泛发生过程中水体含硫致臭物产生情况以及含硫氨基酸转化为 VOSCs 的机制进行研究,主要结论如下:

1) 各类水生生物残体均能诱导湖泛的发生, 其中, 藻源性湖泛发生时水体中 VOSCs 浓度升高尤为突 出, 藻类代谢以及藻体中含有的大量含硫氨基酸可能是这一现象发生的主要原因.

2) 在含硫氨基酸降解产生 VOSCs 的过程中,光照和厌氧条件能有效促进降解的进行, 其中光照的作用 尤为突出.

$3)$ 无论是在生物还是非生物体系中,蛋氨酸降解率与 $\mathrm{NH}_{4}^{+}-\mathrm{N}$ 浓度均存在显著相关性 $(P<0.01)$, 表明 蛋氨酸降解的第一步反应应是脱氨基作用.

4) KMBA 在含硫氨基酸降解体系中难以累积,而 $\alpha$-羊丁酸和 $\alpha$-酮丁酸则有较明显的累积过程,表明脱 氨基作用发生后可能很快即会发生脱甲硫基作用, 且二者有可能同时发生, 由这两种反应生成 MTL, 随后再 生成 DMS、DMDS、DMTS 和 $\mathrm{H}_{2} \mathrm{~S}$ 等. 


\section{4 参考文献}

[ 1 ] 陆桂华,马 倩. 2009 年太湖水域“湖泛”监测与分析. 湖泊科学, 2010, 22(4): 481-487.

[ 2 ] Yang M, Yu J, Li Z et al. Taihu Lake not to blame for Wuxi’s woes. Science, 2008, 319: 158-158.

[ 3 ] 申秋实, 周麒麟, 邵世光等. 太湖草源性“湖泛” 水域沉积物营养盐释放估算. 湖泊科学, 2014, 26(2): 177-184.

[ 4] 刘国锋, 何 俊, 范成新等. 藻源性黑水团环境效应: 对水一沉积物界面处 Fe、Mn、S 循环影响. 环境科学, 2010, 31 (11) : 2652-2660.

[ 5 ] Zhang XJ, Chen C, Ding JQ et al. The 2007 water crisis in Wuxi, China: Analysis of the origin. Journal of Hazardous Materials, 2010, 182(1/2/3): 130-135.

[6] Shen Q, Liu C, Zhou Q et al. Effects of physical and chemical characteristics of surface sediments in the formation of shallow lake algae-induced black bloom. Journal of Environmental Sciences, 2013, 25(12) : 2353-2360.

[ 7 ] Shen Q, Zhou Q, Shang J et al. Beyond hypoxia: Occurrence and characteristics of black blooms due to the decomposition of the submerged plant Potamogeton crispus in a shallow lake. Journal of Environmental Sciences, 2014, 26(2) : 281-288.

[8] 申秋实,邵世光, 王兆德等. 风浪条件下太湖藻源性“湖泛” 的消退及其水体恢复进程. 科学通报, 2012, 57 (12) : 1060-1066.

[ 9 ] Stahl JB. Black water and two peculiar types of stratification in an organically loaded strip-mine lake. Water Research, 1979, 13(5) : 467-471.

[10 ] Duval B, Ludlam SD. The black water chemocline of meromictic Lower Mystic Lake, Massachusetts, USA. International Review of Hydrobiology, 2001, 86(2) : 165-181.

[11] 卢 信, 冯紫艳, 商景阁等. 不同有机基质诱发的水体黑臭及主要致臭物 (VOSCs) 产生机制研究. 环境科学, 2012, $33(9)$ : 3152-3159.

[12］范成新.一种室内模拟水下沉积物再悬浮状态的方法及装置: 中国, 200410014329, 2005.

[13 ] Qin B, Xu P, Wu Q et al. Environmental issues of lake Taihu, China. Hydrobiologia, 2007, 581(1) : 3-14.

[14] Feng Z, Fan C, Huang W et al. Microorganisms and typical organic matter responsible for lacustrine "black bloom”. Science of the Total Environment, 2014, 470: 1-8.

[15] 李学艳, 陈忠林, 沈吉敏等. 固相萃取一气质联机测定水中嗅味物质 2-甲基异茨醇和土霉素. 中国环境监测, 2006, $22(2): 18-21$.

[16] 邵 晨, 黎 雷, 于水利等. 产嗅藻类对东太湖某地原水中嗅味物质 2-MIB 的贡献. 中国环境科学, 2014, 34(9): 2328-2333.

[17] McCarthy TE, Sullivan M. A new and highly specific colorimetric test for methionine. Journal of Biological Chemistry, $1941, \mathbf{1 4 1}(3)$ : 871-876.

[18] Watson SB. Aquatic taste and odor: A primary signal of drinking-water integrity. Journal of Toxicology and Environmental Health-Part a-Current Issues, 2004, 67 (20/21/22) : 1779-1795.

[19] Ginzburg B, Chalifa I, Gun J et al. DMS formation by dimethylsulfoniopropionate route in freshwater. Environmental Science and Technology, 1998, 32(14) : 2130-2136.

[20] Hu HY, Mylon SE, Benoit G. Volatile organic sulfur compounds in a stratified lake. Chemosphere, 2007, 67 (5): 911-919.

[21］李克朗. 太湖蓝藻资源化利用可行性研究 [学位论文]. 无锡: 江南大学, 2009.

[22] Lu X, Fan CX, He W et al. Sulfur-containing amino acid methionine as the precursor of volatile organic sulfur compounds in algea-induced black bloom. Journal of Environmental Sciences, 2013, 25 (1) : 33-43. 